\title{
Aportes de los sistemas locales de justicia a la construcción de una cultura de paz. El caso de la localidad de Kennedy (Bogotá)
}

\author{
Contributions of the Local Justice Systems to the Construction \\ of a Culture of Peace. The Case of Kennedy (Bogotá) \\ Aportes dos sistemas locais de justiça à construção de uma cultura \\ de paz. O caso da localidade de Kennedy (Bogotá)
}

Yesica Alejandra GuZmán-Sossa*

FECHA DE RECEPCIÓN: 15 de OCtUbre de 2019. FeCHA DE APROBACIÓN: 31 DE ENERO de 2019

Doi: https://doi.org/10.12804/revistas.urosario.edu.co/sociojuridicos/a.8346

Para citar: Guzmán-Sossa, Y. A. (2020). Aportes de los sistemas locales de justicia a la construcción de una cultura de paz. El caso de la localidad de Kennedy (Bogotá). Estudios Socio-jurídicos, 22(2), 117-147. Doi: https://doi.org/10.12804/ revistas.urosario.edu.co/sociojuridicos/a.8346

Cuando la espada que usa la justicia, aunque desnuda se conserve casta [...] Cuando el pueblo se encuentre y con sus manos teja él mismo sus sueños y su manta. Cuando de noche grupos de fusiles no despierten al hijo con su habla. Cuando al mirar la madre no se sienta dolor en la mirada y en el alma. Cuando en lugar de sangre por el campo corran caballos y flores sobre el agua. Cuando la paz recobre su paloma y acudan los vecinos a mirarla. Cuando el amor sacuda sus cadenas y le nazcan dos alas en la espalda. Solo en aquella hora podrá el hombre decir que tiene Patria. Carlos Castro SaAvedra

* Este artículo es resultado de la investigación titulada "Aportes de los sistemas locales de justicia a la construcción de cultura de paz. Estudio de caso: localidad de Kennedy, Bogotá", aprobada por el Comité Técnico de Investigaciones de la Facultad de Derecho y Ciencias Políticas de la Universidad de Antioquia a través del Acta 16 de 2017.

** Politóloga de la Universidad de Antioquia, Medellín (Colombia). Docente de la Facultad de Derecho y Ciencias Políticas de la Universidad de Antioquia; integrante del Grupo de Investigación Saber, Poder y Derecho, adscrito a la Universidad de Antioquia. Correo electrónico: yesica.guzman@udea.edu.co. ORCID: https://orcid.org/0000-0002-1231-4627 


\section{RESUMEN}

Se presenta un análisis de los aportes de la implementación de los sistemas locales de justicia a la construcción de una cultura de paz, abordando el caso de la localidad de Kennedy (Bogotá). Se parte de la comprensión de los SLJ como herramienta para garantizar el derecho fundamental de acceso a la justicia y de su relación con la construcción de una cultura de paz. Entre los principales hallazgos se concluye que los SLJ han aportado en la formación de operadores de justicia y en la creación de rutas y espacios de articulación entre actores de la justicia formal y comunitaria, contribuyendo a combatir las barreras operativas y a fortalecer la democracia participativa. Así mismo, se identifican como principales retos para el éxito de la política garantizar su continuidad y sistematicidad en los procesos, lo que implica convertirla en una política de Estado, evitando que esté a merced de la voluntad política, la articulación institucional, la sensibilización y formación continua de los operadores de justicia y la inclusión de actores comunitarios, lo que supondría una democratización de la administración de justicia.

Palabras clave: sistemas locales de justicia, cultura de paz, acceso a la justicia, democracia participativa.

\section{ABSTRACT}

The article presents an analysis of the contributions of the implementation of the local justice systems (SLJ) to the construction of a peace culture, addressing the case of the Kennedy (Bogotá). It is based on the understanding of SLJ as a tool to identify the fundamental right of access to justice and its relationship with the construction of a peace culture. The main findings allow concluding that SLJ has contributed to the training of justice operators and in the creation of routes and spaces for articulation between actors of formal and community justice, contributing to combat operational barriers and strengthening participatory democracy. In the same way, they are identified as the main challenges for the success of the policy, which implies its continuity and systematic processes. It, in turn, means converting it into a state policy, preventing it from being at the mercy of the political will, the institutional articulation, the awareness and continuous training of justice operators, and the inclusion of community actors, which would imply the democratization of the administration of justice.

Keywords: Local systems of justice, culture of peace, access to justice, participatory democracy.

\section{RESUMO}

Se apresenta uma análise dos aportes da implementação dos sistemas locais de justiça à construção de uma cultura de paz, abordando o caso da localidade Kennedy (Bogotá). Parte-se da compreensão dos SLJ como ferramenta para garantir o direito fundamental de acesso à justiça e de sua relação com a construção de uma cultura de paz. Entre os principais resultados se conclui que os SLj têm aportado na formação de operadores de justiça e na criação de rotas e espaços de articulação entre atores da justiça formal e comunitária, contribuindo a combater as barreiras operativas e a fortalecer a democracia participativa. Igualmente, identificam-se como principais desafios para o sucesso da política, garantir sua continuidade e sistematicidade nos processos, o que implica convertê-la em uma política de Estado, evitando que seja dependente da vontade política, a articulação institucional, a sensibilização e formação contínua dos operadores de justiça e a inclusão de atores comunitários, o que suporia uma democratização da administração de justiça.

Palavras-chave: sistemas locais de justiça, cultura de paz, acesso à justiça, democracia participativa. 


\section{Introducción}

Construir una cultura de paz que trascienda el hecho coyuntural de la firma del Acuerdo Final ${ }^{1}$ entre el gobierno colombiano y la entonces guerrilla de las FARC-EP supone lograr que este se arraigue a través de procesos educativos, sociales, culturales, políticos y jurídicos con base en lo local, teniendo en cuenta que es precisamente el contexto territorial, tanto rural como urbano, donde se generaron las dinámicas de continuidad del conflicto armado en Colombia. Así mismo, no es posible la construcción de una cultura de paz sin la satisfacción del derecho de acceso a la justicia, que lleva de manera ínsita la garantía de que todos los derechos son protegidos, y es la forma de evitar que los ciudadanos tomen justicia por su propia mano o acudan a grupos al margen de la ley, haciendo que los conflictos escalen en violencia ante la incapacidad o negligencia del Estado para gestionarlos. No puede haber una cultura de paz allí donde habita la desconfianza en las instituciones a causa de su ineficiencia.

Como una alternativa para la eliminación de las barreras de acceso a la justicia y la posibilidad de construir una cultura de paz en Colombia, surgen los sistemas locales de justicia (SLJ), "una estrategia de organización, coordinación, articulación y cooperación entre los diversos operadores de justicia existentes en el nivel municipal y de estos con la comunidad" (Ardila \& PNUD, 2017a, p. 17). Los SLJ comenzaron a tener fuerza a nivel nacional en el año 2012 en la ciudad de Bogotá, de la mano del programa de casas de justicia y en el marco de la estrategia denominada Sistema Distrital de Justicia, con la intención de propiciar un sistema efectivo en la gestión de conflictos y a su vez cercano al ciudadano.

En el año 2016, en el marco del posacuerdo, el Ministerio de Justicia y del Derecho decidió convertir la iniciativa en una política pública² que, con apoyo de sus socios cooperantes (USAID, Naciones Unidas, Unión Europea), pudiera llegar inicialmente a 167 municipios priorizados, y luego a todo el país (USAID, 2016, p. 7).

Acuerdo Final para la Terminación del Conflicto y la Construcción de una Paz Estable y Duradera (24 de noviembre de 2016, Bogotá, Colombia).

2 Política Pública de Acceso a la Justicia Formal y Alternativa a través de los Sistemas Locales de Justicia. 
Ahora bien, resulta problemático que esta estrategia se replique rápidamente en otros municipios sin haberse aproximado a indagar acerca de los efectos que la implementación de los SLJ ha tenido en la construcción de una cultura de paz, por lo menos en la ciudad de Bogotá, que es el lugar donde tiene mayor trayectoria y donde se sentó el precedente. Esto resulta fundamental si se tiene en cuenta la gran movilidad de esfuerzos y recursos hacia esta estrategia, que exigen velar por su funcionamiento y correspondencia con los fines que declara; además, evaluar su proceso de implementación es el medio para identificar los aspectos por mejorar y los desafíos que se debe prever en su implementación en el resto del territorio nacional.

En virtud de lo anterior, este artículo tiene por objetivo principal identificar los aportes de la implementación de los sistemas locales de justicia a la construcción de una cultura de paz, por medio del estudio de caso de la localidad de Kennedy de Bogotá.

Este artículo es resultado de la investigación titulada: "Aportes de los sistemas locales de justicia a la construcción de cultura de paz. Estudio de caso: localidad de Kennedy, Bogotá"; esta se inscribió en un enfoque teórico institucionalista, en tanto estudiar los SLJ supone abordar las relaciones de cooperación y coordinación entre las instituciones (Losada \& Casas, 2008), su cercanía con los ciudadanos, la formación y participación ciudadana, las transformaciones de las visiones del Estado frente a la administración de justicia, la construcción de nuevas formas de modernización institucional y las políticas públicas.

En coherencia con ello y en términos metodológicos, este estudio se inscribe en un enfoque cualitativo y corresponde a un estudio de caso. Si bien es cierto que un estudio de caso implica atender a su particularidad y concreción, esto no significa que no pueda aportar al análisis del fenómeno en general y tampoco implica que no pueda aportar al estudio de otros casos, teniendo en cuenta que, desde una perspectiva institucionalista, el caso abordado permite entender la influencia del comportamiento de las instituciones y sus relaciones internas en el éxito de una política pública, lo que le da un marco interpretativo más amplio, no como un fenómeno aislado, sino como parte de la discusión actual acerca del proceso de implementación de políticas públicas en el marco del posconflicto. Para el estudio de caso, se diseñaron unos componentes que relacionan 
los elementos fundamentales en la construcción de una cultura de paz y las contribuciones que pueden realizar los SLJ respecto a estos. Para ello se tomaron como referencia los criterios para definir una cultura de paz, planteados en la resolución de la ONU de octubre de 1999, el Manifiesto 2000 por la cultura de paz y no violencia, y los documentos sobre cultura de paz de la Unesco. Estos componentes sirvieron como estrategia para sistematizar, triangular y analizar la información hallada.

Con base en esto, las técnicas utilizadas fueron la revisión documental y la entrevista semiestructurada. La primera, teniendo en cuenta que es un paso necesario para garantizar la rigurosidad y sistematicidad del proceso metodológico; para ello se emplearon como fuentes los documentos institucionales, tales como diagnósticos de la localidad, informes de gestión y los planes de desarrollo. Así mismo, tomando en consideración que se presentaron dificultades en la obtención de datos clave para la investigación, se enviaron derechos de petición a las distintas instituciones partícipes en el proceso de implementación, seguimiento y evaluación de la política.

Por su parte, las entrevistas semiestructuradas sirvieron como herramienta para profundizar en los vacíos existentes identificados en la revisión documental, en tanto el seguimiento a la implementación de esta política no ha sido sistemático, por lo que el cumplimiento del objetivo trazado implicaba insoslayablemente indagar con los actores directamente responsables, en un esfuerzo por documentar y ordenar la información. Se realizaron un total de nueve entrevistas a distintos actores, ${ }^{3}$ entre ellos: operadores de la Casa de Justicia de Kennedy, conciliadores en equidad, jueces de paz, funcionarios de la Personería de Kennedy y de la Secretaría Distrital de Seguridad, Convivencia y Justicia.

Dejando claras las pautas metodológicas que orientaron el abordaje de este objetivo de investigación, se presentan a continuación los resultados. En un primer momento, se aborda el marco conceptual que fundamentó la investigación, pasando por la administración de justicia, la conceptualización de los SLJ y la relación entre cultura de paz y democracia participativa. Seguidamente, se contextualiza el caso explicando

3 Por petición de anonimato de los entrevistados no se presenta en este trabajo el nombre de ninguno de ellos. 
su relevancia en el estudio de los SLJ. Y, finalmente, se presentan los hallazgos de la investigación, señalando los aciertos, puntos críticos y los retos relacionados con la generación de condiciones de posibilidad para el éxito de la política. Este esfuerzo por evaluar el proceso de implementación de los SLj y sus contribuciones en relación con la cultura de paz pretende sentar un precedente para futuras investigaciones sobre el tema y convertirse en un insumo para el análisis de las políticas públicas sobre justicia y construcción de cultura de paz que se viene desarrollando.

\section{Los sistemas locales de justicia como herramienta para garantizar el derecho fundamental de acceso a la justicia y sus aportes a la construcción de una cultura de paz}

\section{Marco general: administración de justicia}

Los sistemas locales de justicia se enmarcan en el concepto de justicia, entendida, para efectos de esta investigación, como "la parte de la función pública que cumple el Estado, encargada por la Constitución Política y la ley, de hacer efectivos los derechos, obligaciones, garantías y libertades para lograr la convivencia social" (Ley 270 de 1996), lo que se conoce precisamente como administración de justicia. De este modo, el sistema jurídico establece derechos y la administración de justicia es el vehículo que permite garantizarlos de manera material, es decir, hacerlos realizables y exigibles (Ardila \& PNUD, 2017a), y es el vehículo para dar satisfacción a las necesidades jurídicas expresadas en los conflictos, esto es, confrontaciones donde participan dos o más actores con posiciones disímiles y que puede poner en vilo los derechos de las partes que están insertas en ellas, más aún si llegan a escalar en violencia.

En esta línea, se puede afirmar que la administración de justicia, en tanto busca atender las necesidades jurídicas, está relacionada íntimamente con la garantía de seguridad a los ciudadanos, que se materializa en el impedir a otros que impongan barreras al ejercicio de los derechos propios, lo que justifica, desde visiones clásicas contractualistas, el surgimiento del Estado y el hecho de que los ciudadanos estén sujetos a él. 
"En Hobbes (la) condición de soberanía se funda en la necesidad de seguridad que reúne a un grupo de hombres alrededor de un órgano de poder mediante un pacto. Los individuos se comprometen a obedecer la voluntad soberana que se expresará a través del Leviatán, de manera general, diciendo qué es justo mediante la ley y, de manera particular, decidiendo las controversias" (Ardila, 2007, p. 164).

Es por esto que la administración de justicia se configura como el marco general de los SLJ, una estrategia que surge con el fin de hacer realizables esas pretensiones, garantizar el derecho fundamental de acceso a la justicia y proporcionar un escenario seguro para el trámite de conflictos. Además, el acceso a la justicia que se busca garantizar se configura como un derecho fundamental estipulado en el artículo 229 de la Constitución Política de Colombia, el cual implica el reconocimiento, respeto de los demás derechos, la equidad en el acceso a ellos y la seguridad de que el ejercicio de estos no será impedido por ninguna persona o agente.

En términos generales, se puede hablar de cuatro tipos de instituciones de administración de justicia: i) la justicia judicial (jurisdicción constitucional, contencioso administrativa y ordinaria); ii) la justicia que ofrece instancias vinculadas a órganos administrativos; iii) los mecanismos alternativos de gestión de conflictos (arbitraje, mediación y conciliación); y iv) la justicia comunitaria (Ardila \& PNUD, 2017a).

\section{Los sistemas locales de justicia como estrategia de eliminación de barreras de acceso a la justicia}

Ahora bien, a pesar de que la oferta de instituciones y mecanismos para administrar justicia es amplia, siguen existiendo barreras de acceso a la justicia, expresadas en: i) barreras culturales y desconocimiento de derechos y enfoques diferenciales, que se hacen visibles en la falta de orientación jurídica, complejidad normativa y ausencia de asistencia legal gratuita; ii) barreras geográficas derivadas de la asimetría en la oferta institucional y el uso limitado de las tecnologías de información y comunicaciones; iii) barreras económicas por el limitado uso del amparo de pobreza, percepción de alto costo y deshonestidad de los abogados; iv) déficit de aceptación y ausencia de reglas en la calidad del servicio; 
v) barreras operativas debido al desorden y complejidad del sistema; y vi) déficit de atención a víctimas y protección a testigos (Corporación Excelencia en la Justicia, 2012).

Es por esto que el Estado ha buscado debilitar estas barreras para garantizar el derecho de acceso a la justicia implementando diversas estrategias, entre ellas los sistemas locales de justicia, que son "una estrategia de trabajo colaborativo entre el Estado y la comunidad para reconocer las necesidades de justicia de cada territorio y asegurar respuestas oportunas y efectivas a los ciudadanos" (Ministerio de Justicia y del Derecho, 2016, p. 2). Es decir, operadores que deciden, operadores consensuales, redes sociales y entidades que impactan sobre la cultura, buscando evitar la frustración del usuario por ausencia de respuestas a sus necesidades reales y el escalamiento de los conflictos que se derivan del sentimiento de abandono, de solo contar con sus propias fuerzas (Ardila E PNUD, 2017b).

Estos sistemas están conformados por diversos actores de la justicia formal, la justicia administrativa y de los gobiernos o administraciones municipales, y por la justicia comunitaria, la justicia propia (de las comunidades afro e indígenas) y la comunidad (Ministerio de Justicia y del Derecho, 2016).

Es así como los SL se estructuran en la agrupación de los actores que, de acuerdo con las dinámicas de conflicto local y la existencia de capacidades instaladas y recursos en el municipio, pueden participar en el desarrollo de labores con diferentes énfasis en la transformación de la cultura de la población frente a la forma de enfrentar conflictos (ya sean instituciones educativas o culturales, comunidades de fe y medios de comunicación). Los vínculos entre los distintos actores del sistema local de justicia son de apoyo, asesoría, remisión (cuando un operador promueve que otro adelante un caso que ha recibido, enviando la documentación y sugiriendo a las partes valerse del otro actor), coordinación y acción conjunta, donde todos los integrantes del sistema se unen para multiplicar su capacidad de impactar en una realidad de mutuo interés (Ardila \& PNUD, 2017d). Igualmente, tienen como base la participación ciudadana activa, situando su papel como relevante no solo en la gestión cotidiana de los conflictos, también en la planeación de estrategias a nivel local, para hacer más efectiva la administración de justicia. 
Esta estructura de los SLj contribuye entonces a simplificar los procesos burocráticos, facilitando la gestión de los conflictos, lo que solo es posible si cada uno de los actores conoce en profundidad sus funciones y las de los demás que integran el sistema, de modo que la remisión y coordinación de los casos pueda ser realmente eficiente.

\section{Funcionamiento del sistema local de justicia}

Los sistemas locales de justicia se instalan por medio de una mesa local o un comité local, donde se reúnen los principales actores: operadores de justicia formal, no formal, comunitaria y, en muchos casos, la comunidad (representada en sus líderes sociales, religiosos, etc.), para tratar los principales conflictos de la localidad, establecer las causas e idear en un trabajo colectivo estrategias para su gestión. Esta mesa construye rutas de manera colectiva, como una herramienta mediante la cual se recogen los acuerdos de trabajo cooperativo entre los actores del sistema y se ponen en manos del público, para que las personas tengan control sobre lo que pueden esperar. También se construyen protocolos de gestión, "una herramienta que permite que entidades públicas, comunitarias y privadas; nacionales y locales; con o sin misionalidad en la administración de justicia, compatibilicen sus tiempos y sus dinámicas para ofrecer una respuesta cooperativa" (Ardila \& PNUD, 2017d, p. 5).

La eficacia del sistema se puede medir en dos escenarios: por un lado, el de los operadores, que cuentan con apoyo para el desarrollo de sus actividades; y, por el otro, el de la comunidad, que ve el sistema como un conjunto articulado de actores que debe responder a sus necesidades de gestión de los conflictos y de amparo de derechos. Es por esto que la materialización de los SLJ requiere que todos los operadores estén dispuestos a ofrecer sus conocimientos y a acoger los de los demás. Este aspecto, aunque parece muy subjetivo, puede potenciarse por medio de la capacitación y sensibilización de los operadores, de modo que comprendan la importancia del rol de cada funcionario en su propia gestión.

De igual forma, la instalación de un sistema local de justicia requiere la apertura de espacios de participación de la comunidad en la toma de decisiones acerca de las estrategias por implementar en la localidad con miras a una efectiva gestión de los conflictos, lo que significa una 
democratización de la administración de justicia en todas sus instituciones, reconocer que la ciudadanía debe ejercer un papel activo a través de la propuesta de soluciones a las barreras de acceso a la justicia que tienen cada uno de los conflictos recurrentes en la localidad.

Por otro lado, los sistemas locales de justicia buscan construir una cultura de paz por medio de la recuperación de la confianza de los ciudadanos hacia las instituciones, lo que solo se logra haciendo más eficiente el sistema judicial, demostrando que es un medio viable para la gestión de conflictos, de modo que los ciudadanos opten por él.

\section{Cultura de paz}

Ahora bien, para comprender los aportes concretos que esta estrategia puede brindar a la construcción y consolidación de una cultura de paz, es preciso comprender antes qué es una cultura de paz y qué implica la construcción de esta. El concepto de cultura de paz se puede explicar con mayor claridad haciendo alusión a su opuesto, la cultura de violencia, porque "si hablamos de cultura de paz como proyecto es porque asumimos cuán enraizada llega a estar en la mayoría de nosotros la cultura de la violencia, entendiendo a ésta última como una forma negativa e inútil de salir de un conflicto" (Fisas, 1998, p. 351 ).

La violencia puede ser definida como "la actitud o el comportamiento que constituye una violación o una privación al ser humano de una cosa que le es esencial como persona (integridad física, moral, derechos, libertades)" (Caireta \& Barbeito, 2005, p. 25). En este sentido, la violencia puede darse espontáneamente, sin que ello implique necesariamente la existencia de un conflicto, sin embargo, casi siempre representa un conflicto que no se logró gestionar correctamente, y que, como consecuencia, se agrava y escala en agresiones. La violencia no es en sí misma una cultura, puesto que la cultura es un conjunto de elementos simbólicos, que constituyen significados, cosmovisiones y costumbres construidos colectivamente, y que se manifiestan en el estilo de vida de las comunidades. "La cultura es, sobre todo, comportamiento cotidiano que refleja la forma de ser de cada cual" (Unesco, 1996, p. 183).

Por lo tanto, para que exista cultura de violencia se establece como criterio fundamental la frecuencia e introyección de esas prácticas, se 
requiere que sea habitual y que a través de ella se generen símbolos y representaciones. La cultura es una construcción social que se da por medio de espacios de gran relevancia social, por ejemplo, la familia y las instituciones educativas, $\mathrm{y}$, en ese sentido, cuando se habla de cultura de violencia se hace alusión a un proceso de aprendizaje de violencia.

A causa de la proliferación de esos aprendizajes y prácticas instaurados en la sociedad como forma inadecuada de afrontar conflictos, surge la necesidad de contraponerla por medio de una cultura de paz. La paz, que en apariencia es un concepto muy abstracto y que siempre ha denotado una condición deseable, se refiere al "proceso de realización de la justicia en los diferentes niveles de las relaciones humanas. Es un concepto dinámico que nos lleva aflorar, afrontar y resolver los conflictos de forma no violenta" (Caireta \& Barbeito, 2005, p. 19), es alli donde encuentra su concreción.

En esta línea, la paz es también la condición, el contexto, para que los conflictos puedan ser transformados creativamente, de tal manera que creamos paz en la medida que somos capaces de transformar los conflictos en cooperación, reconociendo a los oponentes y usando el método del diálogo (Fisas, 1998).

La cultura de paz supone, entonces, desaprender las prácticas de violencia y abrirse a otros caminos para gestionar los conflictos en los distintos territorios. Promueve una cultura que incluya estilos de vida, patrones de creencia, valores y comportamientos que favorezcan la construcción de la paz y acompañe los cambios institucionales que promuevan el bienestar, la igualdad, la administración equitativa de los recursos, la seguridad para los individuos, las familias, la identidad de los grupos o de las naciones, y sin necesidad de recurrir a la violencia (Fisas, 1998, p. 389). Esto es, supone un proceso holístico, que integre todos los aspectos estructurales y organizativos de la sociedad.

Es preciso apuntar que la cultura de paz es un proceso paulatino de transformación social que requiere la participación de todos los sectores y actores, entendiendo la participación en los términos de Sartori (2008) como "tomar parte en persona" (p. 94). Esto implica, por un lado, una voluntad del sujeto, pero, por otro, un diseño institucional que posibilite la inclusión y participación de los ciudadanos; como se señaló anteriormente, requiere un proceso de democratización. 
Así, la construcción de una cultura de paz tiene que ir de la mano con la consolidación de una democracia participativa, entendida como un sistema político donde se da "la incorporación de los sectores marginados del tránsito político, permitiendo que una serie de agentes externos de diversa índole (el Estado, las ONG, la Iglesia, o los voluntariados) pongan en marcha estrategias de capacitación y promoción popular" (Guevara Salamanca, 2009, p. 5). No debe confundirse con la democracia directa, puesto que no pretende abolir la representación, sino dar un papel más activo a los ciudadanos, permitiendo la relación entre nuevos actores e intereses, posibilitando un mayor acercamiento a la institucionalidad, incluso para ejercer control y veeduría sobre ella. De hecho, los actores que deben participar del SLJ son los líderes comunitarios, líderes religiosos, autoridades de instituciones educativas, personas que representan al resto de los habitantes de la localidad.

"La democracia participativa pretende replantear las relaciones sociedad-Estado y pretende la realización plena de la libertad e igualdad de todos los ciudadanos, incluso construye las bases para un nuevo concepto de ciudadanía, basado en el autogobierno, activismo participativo y virtuosidad en el ejercicio de las funciones públicas" (Silva Jiménez, 1997, p. 113).

El vínculo inescindible que fundamenta la relación y codependencia de los procesos de construcción de paz con la democracia participativa parte de la concepción del sujeto como ciudadano, por lo tanto, del escenario donde se sitúa dicha construcción -local e institucional- de paz, a decir, el espacio de lo público, donde "se ponen en discusión cuestiones relativas a la justicia y la vida digna como elementos centrales para repensar la paz, el Estado y la democracia, en definitiva el vivir juntos" (Castaño \& Barrera, 2017, p. 24) y, frente a ello, es condición de posibilidad para el éxito de una sociedad en paz el ejercicio pleno de la ciudadanía respecto a la capacidad de acción, deliberación, control y participación.

Es la ciudadanía organizada la que orienta la agenda pública y el contexto de construcción de paz el que posibilita generar condiciones igualitarias para que la constitución de la agenda pública suceda de esta manera y así poder garantizar que además de finalizar la violencia 
directa entre actores, se faciliten mecanismos y escenarios de incidencia para la superación de las otras violencias presentes en el territorio y que deben ser abordadas como problemas públicos (Castaños $\&$ Barrera, 2017, p. 23).

A menudo se ha reducido la participación política de los ciudadanos al ejercicio del voto, no obstante, es latente la necesidad de que estos participen de todas las funciones públicas del Estado (ejecutiva, legislativa y judicial) y comiencen a tener parte en las decisiones que desde allí se tejen, esta es una condición para la apropiación de esas decisiones y políticas, y es una manera de hacer que las respuestas institucionales sean más pertinentes y no se impongan desconociendo las visiones propias de las comunidades.

Con base en lo anterior, puede afirmarse que una implementación adecuada de los SLJ contribuye a la construcción de una cultura de paz por medio de la democratización de la administración de justicia, esto es, a través de la apertura de espacios donde la comunidad pueda participar en el análisis de las conflictividades a nivel local y en la planeación de estrategias efectivas para la gestión de sus conflictos.

Del mismo modo, los SLJ aportan a la construcción de una cultura de paz a partir de la formación ciudadana, tanto de los operadores como de la comunidad en general, y a la construcción de rutas para el tratamiento efectivo de sus conflictos.

Todas esas acciones promovidas por los SLJ contribuyen a acercar el sistema judicial a la comunidad, de modo que, ante una respuesta efectiva de las instituciones frente a las necesidades jurídicas de los habitantes de la localidad, estos opten por seguir gestionando sus controversias por medio de las instituciones, fomentándose no solo una cultura de la legalidad, sino también una cultura de paz, en tanto se deja de acudir a medios violentos o grupos al margen de la ley para 'resolver' los conflictos.

\section{Componentes de los sistemas locales de justicia en relación con la construcción de cultura de paz}

Conforme con las categorías expuestas, puede deducirse que un sistema local de justicia contribuye a la construcción de cultura de paz 
por medio de diversas acciones expresadas en los subcomponentes de la matriz que se expone a continuación. Con base en ella, se sistematizaron algunos de los hallazgos y se presentan en ese orden los resultados. Es importante aclarar que se habla de 'aportes a la construcción de cultura de paz' porque no puede hablarse de una cultura de paz consolidada en la localidad, sino que los aportes de los SLJ van direccionados a la promoción de la gestión pacífica de conflictos a través de la formación tanto de los operadores de justicia como de la comunidad, y a la potencialización de la participación de actores comunitarios, lo que constituye solo una de las formas de construir cultura de paz, ya que, como se mencionó, es un concepto muy amplio que abarca todos los ámbitos de lo social.

Tabla 1. Aportes de los sistemas locales de justicia a la construcción de una cultura de paz

\begin{tabular}{|l|l|l|}
\hline $\begin{array}{c}\text { Componentes que expresan } \\
\text { aportes de los SLJ a la } \\
\text { construcción de cultura de paz }\end{array}$ & \multicolumn{1}{|c|}{ Subcomponente } & \multicolumn{1}{c|}{ Fuente } \\
\hline $\begin{array}{l}\text { Promoción de la gestión } \\
\text { pacífica de los conflictos } \\
\text { y desarrollo de habilidades } \\
\text { para la gestión de conflictos }\end{array}$ & $\begin{array}{l}\text { 1. Número de operadores } \\
\text { capacitados en los sistemas } \\
\text { locales de justicia. } \\
\text { 2. Número de actividades } \\
\text { de construcción y } \\
\text { divulgación de acuerdos, } \\
\text { rutas y protocolos } \\
\text { interinstitucionales. }\end{array}$ & $\begin{array}{l}\text { Socumentos institucionales } \\
\text { Solicitud de información } \\
\text { a las entidades } \\
\text { correspondientes } \\
\text { Entrevistas semiestructuradas } \\
\text { a operadores }\end{array}$ \\
\hline $\begin{array}{l}\text { Gestión de conflictos } \\
\text { 3. Rutas y protocolos } \\
\text { para la remisión de } \\
\text { casos y comunicación } \\
\text { con otros operadores. } \\
\text { 4. Número de conflictos } \\
\text { remitidos a otras instancias. } \\
\text { 5. Sistemas de información } \\
\text { y seguimiento a los } \\
\text { casos remitidos. }\end{array}$ & $\begin{array}{l}\text { Socumentos institucionales } \\
\text { a las entidades } \\
\text { correspondientes } \\
\text { Entrevistas semiestructuradas }\end{array}$ \\
\hline $\begin{array}{l}\text { Promoción de la participación } \\
\text { democrática: avance en la } \\
\text { implicación de las autoridades } \\
\text { locales y mayor implicación } \\
\text { de la sociedad civil }\end{array}$ & $\begin{array}{l}\text { 6. Actividades de formación } \\
\text { para líderes comunitarios en } \\
\text { sistemas locales de justicia. }\end{array}$ & $\begin{array}{l}\text { Búsqueda documental } \\
\text { Entrevistas semiestructuradas } \\
\text { a operadores }\end{array}$ \\
\hline
\end{tabular}

Fuente: elaboración de la autora. 


\section{Estudio de caso: implementación del SLJ en la localidad de Kennedy de Bogotá}

Teniendo en cuenta estas comprensiones teóricas sobre los sistemas locales de justicia, es muy importante ver cómo han operado en la práctica, de modo que puedan identificarse las fortalezas, límites y aspectos por potenciar en la implementación de esta estrategia. Se escogió el caso de la localidad de Kennedy, partiendo de que fue una de las localidades pioneras en la implementación de estos sistemas en el distrito.

Kennedy es la localidad número ocho de Bogotá y alberga el 14,9\% de la población del distrito. Allí predominan las clases baja y media baja, lo cual genera demandas notorias en la calidad de vida y situaciones de vulnerabilidad propias de este territorio. En el año 2016 se presentaron 26843 casos de violencia interpersonal en Bogotá, de los cuales 4258 correspondieron a la localidad de Kennedy (Bogotá Cómo Vamos, 2017). Así mismo, se ubica en el tercer lugar con mayor número de personas víctimas del conflicto armado, por debajo de Ciudad Bolívar y Bosa (Secretaría de Integración Social, 2017).

Los tres conflictos que predominan en la comunidad son: i) asuntos de familia, ii) cesación en el pago de arriendos y iii) conflictos vecinales. El problema de cesación de pago de arriendos y las demandas por alimentos son muy frecuentes en las personas mayores, que no solo son vulnerables por su condición, sino también porque hay graves niveles de pobreza. La frecuencia de estos conflictos hace que a menudo la demanda en la casa de justicia sea inabarcable (comunicaciones personales, julio de 2018).

Es así como la preocupación por la congestión del sistema ha sido latente en la localidad por el número de habitantes - que cada vez va en ascenso-y por las problemáticas antes enunciadas, lo que la ha llevado a explorar alternativas y a sentar una apuesta clara a la oferta institucional desde la casa de justicia, la cual cuenta con una amplia gama de entidades, como la Comisaría de Familia (Secretaría Distrital de Integración Social), la Casa del Consumidor (Superintendencia de Industria y Comercio), el Consultorio Jurídico, la Defensoría del Pueblo, la Fiscalía General de la Nación, los juzgados civiles, la Oficina de Justicia de Género (Secretaría de la Mujer), la Sijín y la Unidad de Mediación y 
Conciliación. Igualmente, Kennedy es una de las localidades pioneras en la implementación de la figura de los juzgados municipales de pequeñas causas y competencia múltiple en la ciudad de Bogotá ${ }^{4}$ (comunicación personal, julio de 2018).

Kennedy instaló el sistema local de justicia en el año 2015, proceso coordinado por la Secretaría de Gobierno, y, en el año 2016, pasó a ser una estrategia de la naciente Secretaría Distrital de Seguridad, Convivencia y Justicia. Al tiempo se estaban desarrollando otras estrategias, como la formación de 200 candidatos a conciliadores en equidad, así como la actualización de operadores de justicia ya nombrados (funcionario de la Dirección de Acceso a la Justicia de la Secretaría de Seguridad, Convivencia y Justicia, comunicación personal, julio de 2018). "La cohorte comunitaria para los SLJ se completa con la elección de la tercera generación de jueces de paz y reconsideración: 114 líderes elegidos por voto popular para resolver conflictos en las diferentes unidades de planeamiento zonal (UPZ) de Bogotá" (Ardila E PNUD, 2017e, p. 15).

Es preciso señalar que, hasta el momento, han sido instalados 9 sistemas locales de justicia en 10 localidades del distrito: ${ }^{5}$

La administración Petro recoge los acumulados de los gobiernos anteriores y, en un marco de seguridad humana y a tono con los desarrollos que se vienen dando en el nivel nacional del Estado, se compromete a fondo con el establecimiento de nueve sistemas locales de justicia en el que participan operadores de justicia judicial y consensual, entidades públicas responsables del amparo de derechos, organizaciones sociales, medios de comunicación y de la cultura (Ardila \& PNUD, 2017e, p. 13).

La administración de Enrique Peñalosa manifestó la voluntad de asumir el compromiso de dar continuidad a esta política a través de la implementación de estos sistemas en toda la ciudad, como consta en el

4 "El juzgado tiene como función resolver controversias jurídicas, atiende procesos civiles cuyo costo no debe superar la mínima cuantía que equivale a 40 salarios mínimos legales vigentes. En Kennedy se instaura el juzgado de pequeñas causas en el año 2014" (entrevista a juez de pequeñas causas, comunicación personal, julio de 2018).

5 Rafael Uribe Uribe, Teusaquillo, Fontibón, Ciudad Bolívar, Bosa, Kennedy, Santa Fe, Suba, La Candelaria, Usaquén (por su trayectoria, Santa Fe y Candelaria se articulan en un solo SLj) (Ardila \& PNUD, 2017e, p. 14). 
Proyecto Plan de Desarrollo 2016-2020: “Se consolidarán los sistemas locales de justicia en los territorios en los que ya existen y se instalarán diez más en las localidades restantes" (p. 215). Además, plantea su articulación con el Sistema Distrital de Justicia, lo que supondría incrementar el apoyo y la visibilidad de las casas de justicia y las unidades de mediación.

De acuerdo con lo planteado, se realizó una evaluación del proceso de implementación y no de los resultados, puesto que esta etapa no ha concluido y el SLJ aún no se ha consolidado en la localidad.

\section{Análisis de resultados por componente}

\section{Promoción de la gestión pacífica de los conflictos $y$ desarrollo de habilidades para la gestión de conflictos}

Con base en los datos expuestos en la tabla 2, puede evidenciarse que desde el año 2015, cuando se instaló el SLj en la localidad, se han realizado distintas actividades que contribuyen a la formación de los operadores. Uno de los aportes más importantes que se dio al momento de la instalación del SLJ fue la alianza para la promoción de la mediación escolar con 44 instituciones educativas de nivel básico y medio de la localidad, a las cuales se ha seguido vinculando la casa de justicia por medio de talleres y capacitaciones, principalmente en el Colegio INEM y en el Colegio de las Américas.

Esta alianza dio a conocer las acciones y proyecciones del SLJ en la localidad y sirvió de medio para proporcionar a los jóvenes herramientas adecuadas para la gestión de sus conflictos, partiendo del reconocimiento de que el conflicto siempre va estar presente, porque es inherente a la relaciones humanas, pero con la certeza de que la cultura de paz tiene que ver con "la capacidad y habilidad para transformar los conflictos, para que en vez de tener una expresión violenta y destructiva, las situaciones de conflicto puedan ser oportunidades creativas de encuentro, comunicación, cambio" (Fisas, 1998, p. 349). 
Tabla 2. Promoción de la gestión pacífica de los conflictos y desarrollo de habilidades para la gestión de conflictos

\begin{tabular}{|c|c|c|c|c|}
\hline Subcomponente & Número & Dato & Año & Fuente \\
\hline \multirow{2}{*}{$\begin{array}{l}\text { 1. Número de } \\
\text { operadores } \\
\text { capacitados en } \\
\text { los sistemas } \\
\text { locales de } \\
\text { justicia }\end{array}$} & $\begin{array}{l}122 \text { operadores } \\
\text { de justicia de } \\
\text { todo el distrito } \\
\text { (no se obtuvo } \\
\text { dato del número } \\
\text { exacto de los } \\
\text { operadores de } \\
\text { Kennedy) }\end{array}$ & $\begin{array}{l}\text { Diplomado "Estrategias locales } \\
\text { de acceso a la justicia", dirigido } \\
\text { por la Universidad Nacional de } \\
\text { Colombia, en convenio con la } \\
\text { Secretaría de Gobierno Distrital. }\end{array}$ & 2016 & $\begin{array}{l}\text { Entrevista } \\
\text { funcionario } 4 \\
\text { Casa de Justicia } \\
\text { de Kennedy }\end{array}$ \\
\hline & $\begin{array}{l}20 \text { ( } 8 \text { servidores } \\
\text { públicos de } \\
\text { la DAJ; } \\
10 \text { conciliadores } \\
\text { en equidad; } \\
1 \text { juez de paz; } \\
1 \text { mediador } \\
\text { comunitario) }\end{array}$ & $\begin{array}{l}\text { Desarrollo de un proceso de } \\
\text { formación para promover } \\
\text { el fortalecimiento de las } \\
\text { capacidades de los operadores } \\
\text { y otros actores incidentes en el } \\
\text { acceso a la justicia, así como de } \\
\text { todos los equipos territoriales } \\
\text { adscritos a la Dirección } \\
\text { de Acceso a la Justicia. }\end{array}$ & 2017 & $\begin{array}{l}\text { Respuesta a } \\
\text { derecho de } \\
\text { petición envia- } \\
\text { do a la Secre- } \\
\text { taría Distrital } \\
\text { de Seguridad, } \\
\text { Convivencia } \\
\text { y Justicia }\end{array}$ \\
\hline \multirow{3}{*}{$\begin{array}{l}\text { 2. Número } \\
\text { de activi- } \\
\text { dades de } \\
\text { construcción } \\
\text { y divulgación } \\
\text { de acuerdos, } \\
\text { rutas y proto- } \\
\text { colos interins- } \\
\text { titucionales }\end{array}$} & 1 & Mesa local de justicia & 2017 & $\begin{array}{l}\text { Respuesta a } \\
\text { derecho de } \\
\text { petición envia- } \\
\text { do a la Secre- } \\
\text { taría Distrital } \\
\text { de Seguridad, } \\
\text { Convivencia } \\
\text { y Justicia }\end{array}$ \\
\hline & 20 & $\begin{array}{l}\text { Mesas temáticas } \\
\text { locales de justicia }\end{array}$ & 2017 & $\begin{array}{l}\text { Respuesta a } \\
\text { derecho de } \\
\text { petición envia- } \\
\text { do a la Secre- } \\
\text { taría Distrital } \\
\text { de Seguridad, } \\
\text { Convivencia } \\
\text { y Justicia }\end{array}$ \\
\hline & 1 & $\begin{array}{l}\text { Elaboración de una metodología } \\
\text { de elección de los actores } \\
\text { de justicia comunitaria para } \\
\text { promover su participación en las } \\
\text { mesas locales de justicia y en los } \\
\text { respectivos comités temáticos. }\end{array}$ & 2018 & $\begin{array}{l}\text { Respuesta a } \\
\text { derecho de } \\
\text { petición envia- } \\
\text { do a la Secre- } \\
\text { taría Distrital } \\
\text { de Seguridad, } \\
\text { Convivencia } \\
\text { y Justicia }\end{array}$ \\
\hline
\end{tabular}




\begin{tabular}{|c|c|c|c|c|}
\hline Subcomponente & Número & Dato & Año & Fuente \\
\hline & 44 & $\begin{array}{l}\text { Cuando se inauguró el SLJ } \\
\text { se envió una carta a las } 44 \\
\text { instituciones educativas de nivel } \\
\text { básico y medio de la localidad, } \\
\text { para hacer una alianza de } \\
\text { promoción de la mediación } \\
\text { escolar con la casa de justicia } \\
\text { de la localidad. Los colegios } \\
\text { se sumaron a esta iniciativa, } \\
\text { y a partir de allí se ofrecieron } \\
\text { talleres de formación y, además, } \\
\text { se definió que estudiantes } \\
\text { involucrados en conflictos } \\
\text { deben presentarse en la casa } \\
\text { de justicia para gestionarlos y } \\
\text { recibir el acta de acuerdo, sin } \\
\text { la cual no podrán reingresar a } \\
\text { las instituciones educativas. }\end{array}$ & 2015 & $\begin{array}{l}\text { Entrevista a } \\
\text { funcionario } \\
1 \text { de la Casa } \\
\text { de Justicia de } \\
\text { Kennedy }\end{array}$ \\
\hline & 1 & & 2017 & $\begin{array}{l}\text { Entrevista con } \\
\text { funcionaria } \\
1 \text { de la Casa } \\
\text { de Justicia de } \\
\text { Kennedy }\end{array}$ \\
\hline
\end{tabular}

Fuente: elaboración de la autora.

Así mismo, el diplomado ofrecido por la Universidad Nacional de Colombia en el que participaron actores de justicia formal y comunitaria fue muy importante para posicionar los SLJ como una alternativa reconocible para la solución de conflictos; allí se trataron temas como la comprensión integral del conflicto como base del SLJ, la justicia en equidad y los principales aspectos en la constitución de un SLJ. También se abrió la posibilidad dentro del diplomado para la postulación de candidatos a conciliadores en equidad, ofreciendo otro diplomado en administración de justicia en equidad (funcionario de la Casa de Justicia de Kennedy, comunicación personal, julio de 2018).

Estos procesos se consideran un importante aporte a la construcción de cultura de paz, porque contribuyen a la formación de operadores que actúen efectivamente en la protección del derecho de acceso a la justicia de los ciudadanos, de modo que puedan ofrecer un servicio diligente y asertivo frente a las necesidades jurídicas de los individuos, dando como resultado la recuperación paulatina de la confianza en las 
instituciones, consolidando una cultura de la legalidad manifestada en la forma en que deciden tramitar sus conflictos en otras oportunidades.

Ahora bien, hubo varios puntos críticos que arrojaron las entrevistas aplicadas a distintos operadores de justicia en la localidad. Uno de ellos lo puso sobre la mesa un funcionario de la casa de justicia que fue asistente al diplomado dictado en el año 2016; este se refirió a la falta de continuidad en la formación a los operadores y en la promoción de otras actividades que les dieran más herramientas para la gestión adecuada de los conflictos en la localidad.

"A pesar de que me sentí muy motivado por los contenidos del diplomado, que fueron muy buenos y prometedores, siento que solo fue algo circunstancial, que nunca se retomó, esta es la hora en que no estoy enterado de si la instalación del SLJ aún continúa vigente o el proyecto se retiró, pues nunca me avisaron de otra reunión para seguir trabajando" (entrevista a funcionario de la Casa de Justicia de Kennedy, comunicación personal, julio de 2018).

Algo similar ocurrió con la instalación de la mesa local en el año 2017 y las reuniones de las mesas temáticas, como lo indicó uno de los conciliadores en equidad entrevistado:

El año pasado se hizo una reunión, estuvo Personería, Comisaría, los jueces de pequeñas causas, nosotros y los jueces de paz, los de acá, de la casa de justicia, mejor dicho. El compromiso era tratar de seguir haciendo esas reuniones, pero quedó ahí, en esa reunión, y después se citó otra, pero creo que no hubo suficiente convocatoria para que vinieran todos. Se está buscando y siempre hemos buscado construir esa red para remitir. Por ejemplo, por decir algo, de un conflicto local salen otros más, en un tema de reglamento yo capto que hay una niña que está vulnerable o que está siendo violada, entonces yo digo, cómo hago para remitirla. No se ha podido articular de modo que el caso sea exitoso, que no la pongan a voltiar [sic] tanto, no tenemos rutas y la gente queda ahí en la expectativa, uno le ayuda a solucionar con el acuerdo qué hace, pero la otra mitad le queda volando, porque tiene que volver a empezar el trámite y si corre con suerte bien y sino eso queda así, se cansó y lo deja en el camino (conciliador en equidad, comunicación personal, julio de 2018). 
Este aspecto plantea un reto en la implementación de los SLJ: llevar a cabo un proceso continuo no solo de formación, sino también de sensibilización de los operadores, quienes, a pesar de tener un vasto conocimiento en los procedimientos jurídicos, no reconocen la importancia de un trabajo articulado con otros operadores de justicia comunitaria.

Evidencia de lo anterior es que varios jueces de paz de la localidad manifestaron su preocupación frente a la concepción que tienen otros operadores de la justicia formal frente a ellos, en cuanto no los conciben como colaboradores en la descongestión del sistema, sino como trabas a sus acciones y como impedimento para lucrarse económicamente. Esto implicaría que no existe en muchos funcionarios de la justicia formal una conciencia de la importancia de estas figuras de justicia comunitaria, tornándose en un ambiente de rivalidad, representando un obstáculo en la comunicación entre los actores, por ende, en su articulación, y, finalmente, en la implantación del SLj en la localidad. Esto demuestra que las instituciones, si no encuentran canales para resolver los conflictos de intereses de los actores que las constituyen, esto es, no diseñan estrategias de cooperación, pueden convertirse en un serio obstáculo incluso para los proyectos que misionalmente emprenden.

Así, se torna indispensable el retomar las reuniones del comité local y las mesas temáticas, como espacio de articulación por excelencia. Si se dejan intervalos de tiempo tan amplios, difícilmente se podrá consolidar el SLJ en la localidad; además, los conflictos de la población no dan espera y urge pensar estrategias conjuntas para darles tratamiento adecuado.

Por otra parte, también resulta preocupante que no exista aún una figura jurídica mediante la cual se haya reglamentado formalmente la implementación del SLJ, aunque la Secretaría Distrital de Seguridad, Convivencia y Justicia manifestó que se encuentra actualmente en construcción un decreto mediante el cual se institucionalizará el SLJ como política local. Si bien se reconoce que el proceso se está adelantando, resulta crítico que, a tres años de la instalación del comité local, no se cuente con las garantías institucionales para dar permanencia, visibilidad y mayor capacidad vinculante al SLJ, lo que da cuenta de que es una estrategia cuyo éxito está atado a la voluntad política y a las prioridades que esta establezca. Si se tiene la pretensión de que los SLj se consoliden, es preciso que se convierta en una política de Estado y no de gobierno. 
Con relación a la incidencia de la voluntad política, es preciso apuntar que en todas las entrevistas hubo una percepción constante referente al cambio de administración en la alcaldía del distrito, de Petro a Peñalosa; los operadores creen que disminuyeron significativamente los esfuerzos que en materia de implementación del SLJ se estaban adelantando. Se señaló constantemente que desde ese momento los procesos han perdido conexión entre ellos y ha disminuido la frecuencia con que se convocaban, según algunos funcionarios, a causa de que no hubo un adecuado empalme entre las administraciones, percepción que contrasta con la disposición explícita de apoyar esta estrategia, consignada en el Plan de Desarrollo 2016-2020 "Bogotá, mejor para todos".

Por otro lado, partiendo de la identificación de las dificultades de relacionamiento entre los actores de justicia formal y los actores de justicia comunitaria, es necesario que desde la institucionalidad se diseñen estrategias orientadas a generar reflexiones referentes a la forma como se enfrentan los conflictos dentro del sistema de justicia. La construcción de cultura de paz no es un proceso que implica solamente a la comunidad, sino que debe darse desde el interior de las instituciones, desde sus propias formas de organización.

En esta línea, resulta fundamental que los operadores adquieran la conciencia de que son actores claves en la construcción de paz en su localidad y que sus acciones tienen más trascendencia de la que conciben al momento de atender un caso. Para ello, es preciso consolidar una red donde todos se sientan parte, se trata de construir un espacio común (mesa local) con un objetivo común (garantizar el acceso a la justicia a los ciudadanos como fundamento para la construcción de una cultura de paz), un espacio donde se dé una ruptura con los prejuicios frente a los demás operadores, donde se reconozcan las potencialidades de cada figura de justicia formal, informal o comunitaria, tomando como recurso la confianza que hace esperar del otro funcionario reciprocidad, garantizando probabilidades de éxito en la consecución del fin y los medios (rutas) trazados colectivamente. Para ello se requiere constancia en la intervención, citar a talleres y reuniones con una periodicidad definida para la mesa o comité local. 
Yo pienso que es importante consolidar el sistema local de justicia. Sí ha habido, pero la idea es que sea un sistema, como funciona un sistema, digamos que uniendo en cadena de una u otra forma. Nosotros acá siempre hemos funcionado como independientes, por ejemplo. La idea es que cada ente tome conciencia de que hay que trabajar en equipo, ¿quién va a salir beneficiado? Los mismos usuarios y la localidad de Kennedy, que tengamos conocimiento y que todos nos pongamos la camiseta para jalar [sic] del mismo lado (conciliador en equidad, comunicación personal, julio de 2018).

\section{Gestión de conflictos}

Protocolos y rutas de comunicación establecidas para la comunicación entre los operadores

Frente a la construcción de rutas y su promoción para la gestión de conflictos, uno de los funcionarios de la casa de justicia entrevistados manifestó que estas se encuentran en proceso de revisión por parte de la Secretaría Distrital de Seguridad, Convivencia y Justicia, por lo tanto, aún no se han implementado. A continuación, se presenta un esbozo general de estas; a pesar de que no se conoce en detalle y podría sufrir modificaciones, puede evidenciarse que posibilita el hecho de que, ante la incompetencia de una entidad para resolver un conflicto, la remisión de este no quede en manos del ciudadano, que se sentiría agotado y defraudado por el desgaste burocrático y la lentitud de los procesos, sino que estaría a cargo de la casa de justicia, permitiendo una gestión más eficiente.

Las personas llegan primero a registro, donde se les solicita los datos generales de identificación; posteriormente, pasan al CRI, ${ }^{6}$ allí se registra la información referente al tipo de conflicto y se direcciona a la entidad competente; si al llegar a esta el conflicto no puede ser gestionado o solo se le puede dar tratamiento parcial, se remite al consultorio jurídico, donde se estudia el caso y se comunica a los demás operadores correspondientes.

6 Centro de Recepción de la Información. 
Figura 1. Propuesta general de una ruta de atención

de la Casa de Justicia de Kennedy

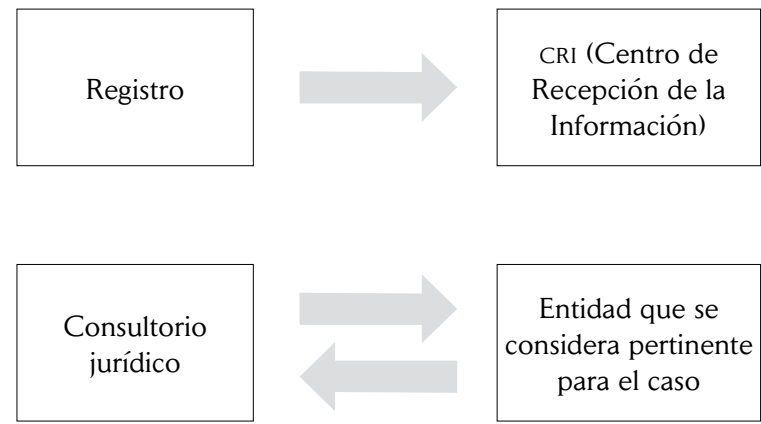

Si el caso no es de

su competencia

Fuente: elaboración de la autora.

El hecho de que se haya formulado esta propuesta de ruta supone en sí mismo un avance y un aporte a la construcción de cultura de paz. Sin embargo, no deja de representar un reto la optimización de los tiempos tanto en la formulación como en la aplicación de estos procesos institucionales, que en ocasiones obstaculizan la puesta en marcha de estas iniciativas. Por lo tanto, se requiere aprobar esta ruta en la mayor brevedad, así como construir un protocolo que la haga efectiva. Este aspecto recobra importancia si se contrasta con la cultura de la inmediatez que ha estado arraigada en la población al ser receptora de propuestas que presentan grupos al margen de la ley para dar salida a los conflictos de manera violenta pero más expedita y ágil. No puede perderse de vista, entonces, que las instituciones tienen la capacidad de generar reglas de juego que produzcan incentivos y que contribuyan a una transformación en la conducta de los ciudadanos, lo que va configurándose como un cambio cultural, en tanto no solo la cultura produce las instituciones, sino que también las instituciones pueden aportar en la construcción de la cultura, en este caso una cultura de paz.

\section{Número de conflictos remitidos a otras entidades $u$ operadores}

Como se observa en la tabla 2, las instituciones a las que se han remitido casos son de tipo formal, esto da cuenta de que en el caso 
de la Personería no hay mayor dificultad para la articulación con estos operadores, no obstante, cabe preguntarse qué sucede con los actores de justicia comunitaria a quienes también llegan casos que tendrían que remitir a la Personería como órgano que vela por la protección y promoción de los derechos humanos - $\mathrm{o}$ a las demás entidades $\mathrm{u}$ operadores-. Aquí se reafirma una vez más la necesidad de potenciar espacios de formación conjunta, donde se reconstruya el imaginario social frente a la validez e importancia de todos los tipos de justicia, en especial la de tipo comunitaria, puesto que entre los operadores de justicia formal existe una mayor comunicación.

Sistemas de información y seguimiento a casos remitidos

Actualmente no existe un sistema de información unificado donde los operadores puedan llevar un registro de los casos que atienden y remiten. No obstante, en la entrevista a la Dirección de Acceso a la Justicia, de la Secretaría Distrital de Seguridad, Convivencia y Justicia, el funcionario aseveró que se encuentra en la etapa de diseño el Sistema de Información Distrital de Justicia (Sidijus), dentro del cual habrá una aplicación denominada Silojus (Sistema de Información de Sistemas Locales de Justicia), en la que se reportaría el seguimiento a la implementación de los SLJ. Estos sistemas de información resultan fundamentales al momento de medir el impacto de la estrategia en cuestión (funcionario de la Secretaría Distrital de Seguridad, Convivencia y Justicia, comunicación personal, julio de 2018).

Así, se presenta como desafío no solo continuar con el diseño e implementación de estos sistemas de información, sino también la debida capacitación a los operadores en el manejo de estas herramientas y el diseño de protocolos institucionales en los que se privilegien medios de comunicación menos etéreos, que permitan conservar un registro, por ejemplo, del uso de correo electrónico en vez de llamadas. Esto con la conciencia de que construir una cultura de paz supone al tiempo reformular las instituciones para la paz, lo que implica que estén diseñadas de modo que puedan garantizar la transparencia de sus acciones y que los ciudadanos puedan ejercer veeduría sobre ellas, lo cual también contribuye al impulso de un mayor compromiso político con la construcción 
de paz, y proporciona las condiciones para que los ciudadanos puedan estar informados y puedan participar de su gestión, contribuyendo de este modo a una consolidación de la democracia participativa.

\section{Promoción de la participación democrática: avance en la implicación de las autoridades locales y mayor implicación de la sociedad civil}

Actividades de formación para líderes comunitarios en los sistemas locales de justicia

Hasta el momento, en Kennedy no se ha dado un proceso concreto de articulación con los líderes comunitarios o actores de la sociedad civil, ni siquiera en un espacio como la mesa local. En la entrevista realizada a un funcionario de la Secretaría de Seguridad, Convivencia y Justicia, este manifestó que están estudiando estrategias para incluir a estos actores prontamente.

Este aspecto constituye uno de los principales retos en la implementación del SLj en Kennedy, pues es indispensable la inclusión de otros actores comunitarios, como líderes sociales, medios de comunicación, representantes de colectivos, los cuales tienen una incidencia en las dinámicas locales, ya que son quienes conocen de primera mano las realidades complejas del territorio. Igualmente, estos actores tienen una vocación de permanencia (a diferencia de los operadores de justicia formal, que tienden a ser trasladados por múltiples causas), y, en ese sentido, son actores claves, porque comunican el sistema con la comunidad en general. A ellos acuden muchas personas para mediar sus conflictos, de modo que, si se garantiza que estos conocen las rutas y están articulados en ellas, podrán orientar a los ciudadanos y acercarlos a mecanismos para el trámite pacífico de sus controversias. Además, como se ha reiterado, no es posible construir una cultura de paz sin la participación de todos los sectores de la sociedad, sin democratizar las instituciones, esto es, que la ciudadanía tome parte activa en la formulación de soluciones eficaces y asertivas frente a las problemáticas locales que ellos conocen en profundidad. 


\section{Conclusiones: fortalezas, puntos críticos y retos en la implementación de los SLJ}

Se ha visto entonces cómo los aportes de la implementación del sistema local de justicia en la localidad de Kennedy para la construcción de una cultura de paz se han enfocado en el fortalecimiento institucional y en la creación de espacios de articulación entre las instituciones administradoras de justicia.

El fortalecimiento institucional se ha dado por medio de la formación de los operadores de justicia en materia de gestión pacífica de los conflictos, brindándoles espacios de capacitación donde pudieran adquirir herramientas teóricas para comprenderlos de manera integral. Esto también tiene un efecto práctico, porque, una vez comprendido el conflicto, se tienen más elementos para dar trámite exitoso a este, disminuyendo las barreras de acceso a la justicia, por ejemplo, el desconocimiento de derechos y enfoques diferenciales.

Así mismo, se ha señalado el diseño de una ruta que podría ayudar a agilizar los procesos burocráticos al momento de gestionar un conflicto, dado que el proceso de remisión estaría garantizado por la casa de justicia en vez de ser una labor que le corresponda al ciudadano, dando mayor facilidad a este y asegurando una mayor eficiencia en los resultados, lo que se traduce en una mayor satisfacción a las necesidades jurídicas de los ciudadanos. Esto contribuye a crear un precedente positivo, de modo que, ante nuevos casos, la persona opte por resolver sus conflictos por estos medios institucionales y no a través de la violencia o intermediarios ilegales, transformando paulatinamente la cultura de violencia que les lleva a acudir a medios no institucionales ni pacíficos. Además, contribuiría a disminuir las barreras operativas, debido al desorden y complejidad del sistema, y las barreras económicas referidas a los altos costos.

Ahora bien, en este punto se presenta un reto que no puede evadirse si se quiere garantizar el desarrollo de los SLJ, este consiste en construir las próximas rutas con la colaboración de los demás actores de la justicia formal, informal, y, sobre todo, con la comunidad, teniendo en cuenta que estas rutas pueden ser un medio para reconstruir el tejido social en la localidad. La cultura es una construcción social y, en ese sentido, 
requiere de un trabajo conjunto para lograr que la comunidad sienta esas rutas y herramientas como propias y no como algo ajeno o impuesto.

En esta misma línea, los espacios de articulación entre las instituciones administradoras de justicia y la comunidad se enfrentan a un reto similar: articular actores comunitarios y construir una conciencia frente a la importancia de las figuras de justicia comunitaria. Los SLJ deben convertirse en un puente entre la ciudadanía y las instituciones, no para que los ciudadanos tengan un rol pasivo de simples receptores de las respuestas institucionales, sino para que posean un papel activo, donde trabajen en la construcción de rutas y herramientas para la consolidación de una cultura de paz.

"La construcción de cultura de paz es un esfuerzo multidisciplinar para transformar desde la educación las concepciones y asumir compromisos con la finalidad de originar la presencia de la paz en la cultura, comprendiendo que esta tarea de construir debe trascender los límites de los conflictos, para convertirse en vivencia cotidiana de contextos como la escuela, el trabajo, la familia, el Estado, la sociedad civil, entre otros" (Hernández, Luna \& Cadena, 2017, p. 152).

Es por todo lo anterior que se recomienda la apertura de los espacios ya instaurados, tales como el comité local y las mesas temáticas para la participación de los líderes más activos en la localidad, pues ello podría contribuir a: i) construir un diagnóstico de conflictividades más amplio y profundo, que serviría de insumo para pensar y proponer estrategias concretas y focalizadas; ii) fortalecer la red colaborativa y, de ese modo, hacerla más efectiva, ya que entre más actores participen de ella hay más probabilidades de canalizar conflictos con los que los operadores tradicionales no llegan a tener contacto, es decir, se amplía la cobertura y capacidad del SLJ. Esto implica también, como se ha señalado, una democratización de la administración de justicia.

La seguridad y un adecuado acceso a la justicia, en condiciones de calidad y oportunidad, lo mismo que la protección de los derechos humanos y la reparación a las víctimas, son elementos centrales para garantizar la prevalencia del Estado de derecho en un escenario de paz y posconflicto. En efecto, profundizar el sistema democrático y consolidar una paz estable y duradera exige romper estos obstáculos y avanzar hacia 
la construcción de un Estado más efectivo (Departamento Nacional de Planeación, Consejo Nacional de Política Económica y Social, 2016).

Ahora bien, este problema de desarticulación institucional no es exclusivo del caso de Kennedy, es más bien un problema de orden nacional. En este sentido, analizar este caso es apenas un primer esfuerzo, pero absolutamente necesario, consistente en identificar a partir de un caso - que lleva más trayectoria en comparación con el resto- esas dificultades en el interior de las instituciones, derivadas de conflictos de intereses particulares que cristalizan a su vez la falta de conciencia de los servidores públicos respecto a sus funciones, su responsabilidad y capacidad de impacto. Kennedy es solo la expresión de una visión de la justicia comunitaria que debe transformarse desde las prácticas cotidianas, a partir de un proceso formativo de los actores de la justicia formal, de la democratización de las instituciones encargadas de administrar justicia, que, como se ha mencionado reiterativamente, requiere la inclusión de los actores comunitarios y el reconocimiento de los aportes que, desde su experiencia y conocimiento directo de las realidades de las localidades o municipios, pueden brindar.

Este caso reafirma entonces la capacidad que tienen las instituciones para transformar la cultura, para agenciar el cambio, para incentivar a las comunidades y para, finalmente, demarcar el rumbo, el éxito o el fracaso de las políticas públicas. El contexto del posacuerdo requiere una voluntad política-institucional para reconstruir y abrir nuevas rutas para la gestión del conflicto, que no pase por alto las particularidades contextuales de cada territorio, rutas que posibiliten un trabajo conjunto, una construcción colectiva y una apropiación del cambio.

Son muchos los retos que deben asumirse en la implementación de los SLJ en la localidad y en el resto del territorio nacional, pero esencialmente se requiere voluntad política para la ejecución de estos. La construcción de una cultura de paz no debe dejar de ser la aspiración de los gobiernos y debe materializarse a través de instituciones fuertes que los ciudadanos perciban como transparentes y efectivas; en este sentido, es necesario potenciar este tipo de estrategias encaminadas al logro de estos objetivos desde el nivel local hasta el nivel nacional. 


\section{Referencias}

Ardila, E. A. (2007). Breve historia de un eclipse. Pensamiento Jurídico, (20).

Ardila Amaya, E., E PNUD. (2017a). Vol. 1. ¿Qué es y para qué sirve un sistema local de justicia? Bogotá: PNUD.

Ardila Amaya, E., \& PNUD. (2017b). Vol 2. ¿Cómo se implementan los sistemas locales de justicia? Bogotá: PNUD.

Ardila Amaya, E., E PNUD. (2017c). Vol. 3. Cercanía, seguridad y calidad de vida: las respuestas del sistema local de justicia. Bogotá: PNUD.

Ardila Amaya, E., G PNUD. (2017d). Vol. 4. Así trabaja un sistema local de justicia. Bogotá: PNUD.

Ardila Amaya, E., E PNUD. (2017e). Vol. 6. ¿Cómo le va a Bogotá con los sistemas locales de justicia? Bogotá: PNUD.

Bogotá Cómo Vamos. (2017). Informe de calidad de vida en Bogotá 2016. Recuperado de https://bogotacomovamos.org/interactivo-informe-de-calidad-de-vida-2016/

Caireta Sampere, M., \& Barbeito Thonon, C. (2005). Cuadernos de educación para la paz. Introducción de conceptos: paz, violencia, conflicto. Barcelona: Universidad Autónoma de Barcelona. Recuperado de http://escolapau.uab. cat/img/programas/educacion/publicacion002e.pdf

Castaño Torres, S., \& Barrera Yate, D. (2017). La construcción de paz como posibilidad de profundización de la democracia. Neiva: Universidad Surcolombiana.

Colombia, Ministerio de Justicia y del Derecho. (2016). Hablemos sobre los sistemas locales de justicia. Organización y competencias de autoridades judiciales y administrativas en la resolución de conflictos a nivel local. Cartilla para operadores de justicia local. Bogotá: Ministerio de Justicia y del Derecho.

Colombia, Departamento Nacional de Planeación, Consejo Nacional de Política Económica y Social. (2016). Documento Conpes 3867 de 2016, “Estrategia de preparación institucional para la paz y el posconflicto".

Colombia, Secretaría de Integración Social. (2017). Acercamiento al comportamiento de las violencias en Kennedy. Año 2015-2016. Bogotá. Documento inédito.

Corporación Excelencia en la Justicia. (2012). Necesidades jurídicas insatisfechas. Bogotá: Fondo de Cultura Económica.

Fisas, V. (1998). Cultura de paz y gestión de conflictos. París: Icaria Editorial, S.A. Guevara Salamanca, J. D. (2009). La democracia participativa como instrumento de dominación de la esfera pública. Una mirada crítica a la realidad política colombiana a partir de la Constitución de 1991. Bogotá: Universidad Colegio Mayor Nuestra Señora del Rosario. 
Hernández Arteaga, I., Luna Hernández, J. A., \& Cadena Chala, M. C. (2017). Cultura de paz: una construcción desde la educación. Revista Historia de la Educación Latinoamericana. Recuperado de http://www.scielo.org.co/pdf/ rhel/v19n28/v19n28a09.pdf

Losada, R., \& Casas Casas, A. (2008). Enfoques para en análisis politico. Bogotá: Pontificia Universidad Javeriana.

Sartori, G. (2008). ¿Qué es la democracia? México: Taurus.

Silva Jiménez, A. M. (1997). Hacia una democracia participativa (II parte). Teoría participacionista de la democracia. Revista de Derecho, 8(1), 113-122. Unesco. (1996). From a culture of violence to a culture of pace. Paris.

USAID. (2016). Documento de Politica Pública de Acceso a la Justicia Formal y Alternativa a través de los Sistemas Locales de Justicia. USA: Programa Colombia Transforma. 